\title{
Trajectory Optimisation for Avionics-Based GNSS Integrity Augmentation System
}

\author{
Roberto Sabatini ${ }^{1}$, Terry Moore ${ }^{2}$, Chris Hill ${ }^{2}$, Alessandro Gardi ${ }^{1}$, Subramanian Ramasamy ${ }^{1}$ and Michael Gilgien ${ }^{3}$ \\ ${ }^{1}$ School of Engineering, RMIT University, \\ Melbourne, VIC 3000, Australia \\ ${ }^{2}$ Nottingham Geospatial Institute, University of Nottingham, \\ Nottingham, NG7 2TU, UK \\ ${ }^{3}$ Department of Aerospace Engineering, Cranfield University \\ Cranfield, MK43 0AL, UK \\ roberto.sabatini@rmit.edu.au
}

\begin{abstract}
In this paper, trajectory optimisation algorithms developed specifically for the Global Navigation satellite System (GNSS) Avionics-Based Integrity Augmentation (ABIA) system are presented. The ABIA system is designed to increase the levels of integrity and accuracy (as well as continuity in multi-sensor data fusion architectures) of GNSS in a variety of mission- and safetycritical aviation applications. The trajectory optimisation algorithms can be employed both for flight planning as well as realtime optimisation of manned and unmanned aircraft for all flight phases. Three and six Degrees-of-Freedom (3-DoF/6-DoF) aircraft dynamics models are adopted to generate a number of feasible flight trajectories that also satisfy the GNSS constraints. A detailed simulation case study is presented to evaluate the performance of trajectory optimisation algorithms for GNSS integrity augmentation using an AIRBUS A320 3-DoF aircraft dynamics model. Results confirm that the employed trajectory optimisation algorithms are capable of supporting high-integrity tasks when GNSS is used as the primary source of navigation data.
\end{abstract}

Keywords-Avionics Based Integrity Augmentation, Trajectory Optimisation, Caution and Wanirng Flags, GNSS Integrity, Missionand Safety-Critical GNSS Applications.

\section{INTRODUCTION}

Trajectory optimisation algorithms for route planning and real-time re-planning are key enablers of the Air Traffic Management (ATM) modernisation as proposed by SESAR/NextGen programmes [1, 2]. The optimisation process will be an integral part of the real-time trajectory negotiation and validation paradigm between aircraft and ATM organisations. This will require on-board trajectory optimisation by Next Generation Flight Management Systems (NG-FMS), as well as ground trajectory optimization by the air traffic controllers $[3,4]$. Requirements in term of real-time capability and computational power are different depending on the situation. In recent years, a number of trajectory optimisation techniques and methods have been developed for aviation applications. In particular direct methods using pseudospectral discretization techniques are found to be very accurate, with a high convergence rate. Even exponential if the problem is smooth. Hence pseudospectral optimization appears to be well suited for avionics and ATM implementations [5].

Global Navigation Satellite Systems (GNSS) are key players in modern aircraft navigation. The introduction of GNSS in the 1990s allowed the aviation community to initiate a gradual transition from sensor-based navigation to Performance-Based Navigation (PBN) concepts [6]. Notwithstanding, GNSS still suffers from signal degradation and outages due to a number of factors including antenna obscuration, bad satellite geometries, low Carrier-to-Noise ratios $\left(\mathrm{C} / \mathrm{N}_{0}\right)$, Doppler shifts, interference and multipath effects. Some of these are well mitigated using differential GNSS techniques with Space or Ground Based Augmentation Systems (SBAS, GBAS). But there is little to do against user segment related integrity degradations [7]. Antenna masking, bad satellite geometry, multipath and low signal-tonoise ratio degradations mostly depend on current flight parameters of the receiver (position, Euler angles, velocity), which in turn are influenced by operational or environmental conditions.

Nevertheless, suitable Aircraft Based Augmentation Systems (ABAS) implementing avionics-based integrity monitoring and augmentation features as proposed in [8, 9] is capable of timely detecting and even predicting the onset of these degradations. Based on these results, a mitigation of these degradations by flying appropriately designed trajectories is a very effective solution that can be integrated in current avionics and ATM systems. The proposed Avionics Based Integrity Augmentation (ABIA) systems can be integrated in an onboard Flight Management System (FMS) and enhance the embedded trajectory planning and real-time re-planning functionalities to avoid integrity degradations. 
The objective of the trajectory optimisation functionality presented here is thus to determine the best possible trajectory considering the capability of the aircraft and its mission profile. The main goal is improving efficiency in terms of operational and economic/environmental indicators, but some other considerations need to be taken into account during the optimisation process including ATM separation and sequencing, noise sensitive areas and airspace restrictions. An optimisation of aspects related to safety can be also envisaged, especially for safety-critical phases of flight such as precision approach and landing. The safety requirements for these phases involve avionic systems certification up to a certain level depending on their capacity, but to attain higher levels of safety supporting fully automated zero visibility landing (CAT IIIc) and to meet the signal-in-space integrity requirements, the previously mentioned GNSS degradations must be successfully addressed. Hence trajectory optimisation pursuing the avoidance of GNSS integrity degradations can allow significant increases in the levels of safety for safety- and mission-critical operation.

\section{ABIA SYSTEM}

As pointed out earlier, the following conditions cause navigation data outages or severe performance degradations: antenna obscuration, bad satellite geometries and low Carrier-toNoise ratios $\left(\mathrm{C} / \mathrm{N}_{0}\right)$, Doppler shifts, interference and multipath [8-10]. The last two problems could be mitigated by existing technology solutions (i.e., by suitable selection of VHF/UHF data links, filtering the radio frequency signals reaching the GNSS antenna, identifying suitable locations for the GNSS antenna, etc.). However, to prevent critical events during particular approach procedures (e.g., curved and segmented approaches) as well as in upcoming safety-critical applications such as Sense-and-Avoid (SAA), other factors causing GNSS degradations or losses have to be addressed carefully. Therefore the ABIA system is conceived to alert the pilot or the remote pilot in an Unmanned Aircraft System (UAS) context when the critical conditions for GNSS signal loss are likely to occur (within a specified maximum time-to-alert). The aircraft onboard sensors supply information on relevant aircraft flight parameters (navigation data, engine settings, etc.) to an Integrity Flag Generator (IFG), which is connected to the on-board GNSS receiver. Specific mathematical algorithms have been developed to cope with the main causes of GNSS signal outages and degradation in flight, namely: obscuration, multipath, interference, fading due to adverse geometry and Doppler shift $[8,9]$. Adopting these algorithms, the ABIA system can provide steering information to the pilot and electronic commands to the flight control system, allowing real-time avoidance of safetycritical flight conditions and fast recovery of the required navigation performance in case of GNSS data losses. This is achieved by implementing both caution (predictive) and warning (reactive) integrity flags, as well as 4-Dimensional Trajectory (4DT) optimisation models suitable for all phases of flight.
Therefore, as shown in Fig. 1, in the advanced ABIA system discussed here on-board sensors provide information on the relevant aircraft flight parameters (navigation data, engine settings, etc.) to an Integrity Flag Generator (IFG), which is also connected to the GNSS system. Using the available data on GNSS and the relevant flight parameters, integrity signals are generated to be used by a trajectory optimisation/Flight Path Optimisation (FPO) module or to be sent to the UAS Ground Control Station (GCS). This advanced ABIA system addresses both the predictive and reactive nature of GNSS integrity augmentation by producing suitable integrity flags (cautions and warnings) in case of predicted/ascertained GNSS data losses or unacceptable signal degradations exceeding the Required Navigation Performance (RNP) specified for each phase of flight, and providing guidance information to the remote pilot/autopilot to avoid further data losses/degradations.

To achieve this, the Integrity Flag Generator (IFG) module produces the following integrity flags $[8,9]$ :

- Caution Integrity Flag (CIF): a predictive annunciation that the GNSS data delivered to the avionics system is going to exceed the RNP thresholds specified for the current and planned flight operational tasks (alert status).

- Warning Integrity Flag (WIF): a reactive annunciation that the GNSS data delivered to the avionics system has exceeded the RNP thresholds specified for the current flight operational task (fault status).

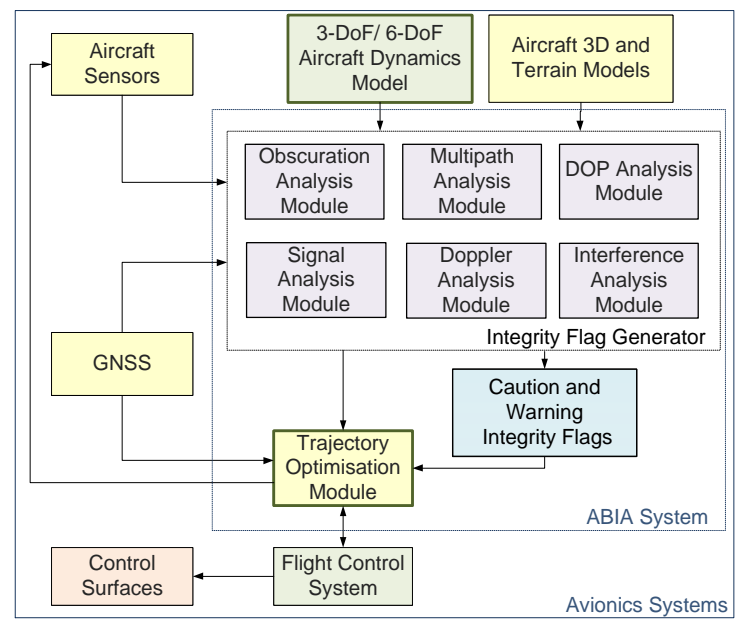

Fig. 1. ABIA system architecture.

The following definitions of Time-to-Alert (TTA) are applicable to the ABIA system [8, 9]:

- ABIA Time-to-Caution (TTC): the minimum time allowed for the caution flag to be provided to the user (pilot/remote pilot/FPO module) before the onset of a GNSS fault resulting in an unsafe condition. 
- $\quad$ ABIA Time-to-Warning (TTW): the maximum time allowed from the moment a GNSS fault resulting in an unsafe condition is detected to the moment that the ABIA system provides a warning flag to the user (pilot/remote pilot/FPO module).

\section{TRAJECTORY OPTIMISATION ALGORITHMS}

All the standard components of an optimisation problem including GNSS constraints are considered in this implementation. A flight dynamics model of the aircraft provides the dynamic constraints and allows generating a trajectory that is flyable by the aircraft. Integrity degradations and current GNSS parameters define a certain number of path constraints. They ensure that integrity degradations will be avoided on all flight phases. Boundary conditions are set including minimal, maximal, initial and final values for the entire state and command variable. They are dictated by the aircraft sensors, which supply current flight phase parameters, and by the FMS, which supplies the information about the mission profile from the flight plan. All the necessary constraints for integrity degradations are included in the form of path constraints. This choice is made for simplicity and because only integrity navigation optimization is considered in this research, but more complex criteria can be set based on aircraft performances.

The IFG outputs (CIFs and WIFs) are used in the FPO module. The key requirement of the FPO module is to generate guidance information that are used to optimise the short-term Autopilot and Flight Director System (A\&FDS) control parameters (e.g., attitude angles and airspeed), as well as the medium/long-term trajectory to be flown. The information update process starts from the current information stored in the FMS and produces a new set of geometric/dynamic constraints in all conditions where CIFs are raised (to avoid the subsequent rise of WIFs). A trajectory optimisation process is then initiated to achieve specified mission objectives and to comply with this new set of constraints. The results obtained from the computation of the optimal trajectory can be utilised as new inputs to the IFG software (both in real-time and missionplanning implementations), so further updates can be performed when needed to prevent the triggering of CIFs and WIFs.

From a practical point of view, trajectory optimisation can be defined as the action of finding the inputs to a given system characterised by a set of equations of motion and dynamic constraints, which will maximise or minimise specific parameters (e.g., time, fuel consumption, distance from another aircraft, relative velocities). In most cases, optimisation problems cannot be solved analytically and, therefore, numerical iterative methods need to be used. One of the key challenges of the online trajectory optimisation task is to produce results in real-time (real-time here is intended for the specific application/scenario involved), since the mathematical algorithms and the associated numerical solvers have to be capable of producing accurate and usable outputs in a relatively short time. Offline and online aircraft trajectory optimisation tasks are typically formulated as Optimal Control Problems (OCP). This is because optimal control theory provides a wellestablished framework for the determination optimal trajectories of dynamical systems (in a mathematical sense). In the OCP formulation, the trajectory optimisation problem can be analytically stated as follows:

"Determine the states $x(t) \in \mathbb{R}^{n}$, the controls $u(t) \in \mathbb{R}^{m}$, the parameters $p \in \mathbb{R}^{q}$, the initial time $t_{0} \in \mathbb{R}$ and the final time $t_{f} \in \mathbb{R} \mid t_{f}>t_{0}$, that optimise the performance index [10]:

$$
J=\Phi\left[\mathbf{x}\left(t_{f}\right), \mathbf{u}\left(t_{f}\right), \mathbf{p}\right]+\int_{t_{0}}^{t_{f}} \Psi[\mathbf{x}(t), \mathbf{u}(\mathrm{t}), \mathbf{p}] d t
$$

subject to the dynamic constraints:

$$
\dot{\mathbf{x}}(\mathrm{t})=\mathbf{f}[\mathbf{x}(\mathrm{t}), \mathbf{u}(\mathrm{t}), \mathrm{t}, \mathbf{p}]
$$

to the path constraints:

$$
\mathbf{C}_{\min } \leq \mathbf{C}[\mathbf{x}(\mathrm{t}), \mathbf{u}(\mathrm{t}), \mathrm{t} ; \mathbf{p}] \leq \mathbf{C}_{\max }
$$

and to the boundary conditions:

$$
\mathcal{B}_{\min } \leq \mathcal{B}\left[\mathbf{x}\left(\mathrm{t}_{0}\right), \mathbf{x}\left(\mathrm{t}_{\mathrm{f}}\right), \mathbf{u}\left(\mathrm{t}_{0}\right), \mathbf{u}\left(\mathrm{t}_{\mathrm{f}}\right) ; \mathbf{p}\right] \leq \mathcal{B}_{\max }
$$

where $\Phi$ is the Mayer term and $\Psi$ is the Lagrange term of the cost function expressed in a Bolza form. OCP can be solved using various methods and the two main categories are: direct methods and indirect methods. The indirect methods are based on calculus of variation. The problem is transcribed in a Boundary Value Problem (BVP) that needs maximum and minimum boundaries and an initial guess also accounting for costate. Indirect methods for optimisation present some advantages since the number of variables is not extensive as in the case of direct methods, so that computational requirements are reduced. However, the involved Hamiltonian dynamics are often unstable and the error with unknown initial conditions grows rapidly. If the initial guess differs widely from the optimal solution, the system will converge slowly or even diverge in some cases. Therefore, in the case of complex problems, a more appropriate method must be employed.

In the case of direct methods, the aircraft dynamics (continuous) variables are translated into a number of discretised state and command parameters. The problem is then transformed into a finite-dimensional non-linear optimisation problem and the states are implicitly integrated with a quadrature method for a subset of points (nodes) chosen in the main interval defined by the maximum and minimum boundaries. Direct methods are further divided into direct shooting and collocation methods. Direct shooting methods employ user defined analytical 
functions to parameterise the controls for the entire OCP domain, while collocation methods adopt piecewise polynomial interpolating functions to parameterise states and control. The most computationally efficient class of collocation methods adopts a basis of linearly independent polynomial functions and is called pseudo-spectral. The main drawbacks of direct methods are a strong dependency on the accuracy of the selected discretisation functions and the large number of variables introduced by the parameterisation of states and controls, as well as by the implicit integration of subintervals. Therefore, the computational power required for direct methods can be significant. This problem has often being solved considering that the Jacobian and Hessian of the constraints comprise a majority of null derivatives.

Compared with indirect methods, in the direct methods there are no means to know how close a given solution is to the optimal solution, which instead is possible in the case of indirect computation. Planning or optimising a new trajectory for an aircraft is subject to numerous objectives and constraints. Those can be derived from ATM imposed requirements, flight plan/mission objectives, Separation Assurance and Collision Avoidance (SA\&CA), and environmental requirements. Thus, the optimisation process needs to find the best trade-off between all objectives subject to the dynamics/operational constraints associated with the platform, the planned mission and the current flight profile/phase. Clearly, different sets of data and significantly different objectives/constraints can be used at the mission planning stage and in real-time flight trajectory optimisation tasks.

ABIA offers the advantage of meeting the requirements of strategic and tactical air operation tasks, with a possibility to also enhance the performance of SA\&CA systems that rely on GNSS as the primary source of navigation data. These include modern cooperative SA\&CA systems (e.g., ADS-B/TCAS) or non-cooperative sensors integrated with GNSS-driven Guidance, Navigation and Control (GNC) systems. For a practical implementation, some additional considerations have to be made considering the following aspects. The new trajectory determined by the FPO module has to be completely flyable by the aircraft and the mission (defined in the active FMS flight plan) shall not be compromised by the new trajectory. Additionally, the new trajectory shall not lead to other hazards like terrain, traffic or weather. As a consequence, the FPO module has to be designed to allow the dynamic setting of path constraints and boundary conditions for the entire set of variables involved. From the discussion above the following key requirements are derived:

The FPO module shall react to any CIF/WIF triggering as follows:

- Initiating an early-correction loop that prevents the occurrence of a WIF, as soon as CIF is generated.
- Initiating an immediate (emergency) correction in the unlikely event of a WIF not preceded by a CIF.

As soon as activated, the FPO module shall set dynamic constraints allowing computation of an optimal flight trajectory that prevents the triggering of new CIFs/WIFs and that minimises the deviations from the original aircraft trajectory (e.g., FMS flight plan).

\section{Dynamics Model}

Assuming the aircraft to be a rigid body with a static mass distribution, an accurate model of its flight dynamics can be introduced, which complement the equilibrium of forces along the coordinate axes of a suitable Cartesian reference frame located in the centre of mass of the aircraft, named body frame, with the equilibrium of their momentums. This model involves a high number of parameters to define the properties of inertia and of aerodynamic stability and control forces. Adequate experimental and numerical investigations are typically required in order to define the parameters with good precision. For the implementation in TOP, and for other applications including flight simulation and trajectory estimation, flight dynamics are typically transcribed in a set of Differential Algebraic Equations (DAE) [11]. The set of DAE and complementary kinematic relations defining the six Degree of Freedom (6-DoF) rigid body dynamics of a fixed-wing aircraft are [12]:

$$
\begin{aligned}
& {\left[\begin{array}{c}
\dot{\mathrm{u}} \\
\dot{\mathrm{v}} \\
\dot{\mathrm{W}}
\end{array}\right]=\frac{\mathrm{g}}{\mathrm{W}}\left[\begin{array}{c}
\mathrm{F}_{\mathrm{X}_{\mathrm{b}}} \\
\mathrm{F}_{\mathrm{Y}_{\mathrm{b}}} \\
\mathrm{F}_{\mathrm{Z}_{\mathrm{b}}}
\end{array}\right]+\mathrm{g}\left[\begin{array}{c}
-\mathrm{s} \theta \\
\mathrm{s} \phi \mathrm{c} \theta \\
\mathrm{c} \phi \mathrm{c} \theta
\end{array}\right]+\left[\begin{array}{c}
\mathrm{rv}-\mathrm{qw} \\
\mathrm{pw}-\mathrm{ru} \\
\mathrm{qu}-\mathrm{pv}
\end{array}\right]} \\
& {\left[\begin{array}{c}
\dot{\mathrm{p}} \\
\dot{\mathrm{q}} \\
\dot{\mathrm{r}}
\end{array}\right]=\left[\begin{array}{ccc}
\mathrm{I}_{\mathrm{XX}_{\mathrm{b}}} & 0 & -\mathrm{I}_{\mathrm{XZ}_{\mathrm{b}}} \\
0 & \mathrm{I}_{\mathrm{YY}_{\mathrm{b}}} & 0 \\
-\mathrm{I}_{\mathrm{ZX}_{\mathrm{b}}} & 0 & \mathrm{I}_{\mathrm{ZZ}_{\mathrm{b}}}
\end{array}\right]^{-1} \text {. }} \\
& \cdot\left[\begin{array}{c}
\mathrm{M}_{\mathrm{X}_{\mathrm{b}}}+\left(\mathrm{I}_{\mathrm{YY}_{\mathrm{b}}}-\mathrm{I}_{\mathrm{ZZ}_{\mathrm{b}}}\right) \mathrm{qr}+\mathrm{I}_{\mathrm{XZ}} \mathrm{pq} \\
\mathrm{M}_{\mathrm{Y}_{\mathrm{b}}}+\left(\mathrm{I}_{\mathrm{ZZ}_{\mathrm{b}}}-\mathrm{I}_{\mathrm{XX}_{\mathrm{b}}}\right) \mathrm{pr}+\mathrm{I}_{\mathrm{XZ}_{\mathrm{b}}}\left(\mathrm{r}^{2}-\mathrm{p}^{2}\right) \\
\mathrm{M}_{\mathrm{Z}_{\mathrm{b}}}+\left(\mathrm{I}_{\mathrm{XX}_{\mathrm{b}}}-\mathrm{I}_{\mathrm{YY}_{\mathrm{b}}}\right) \mathrm{pq}-\mathrm{I}_{\mathrm{XZ}_{\mathrm{b}}} \mathrm{qr}
\end{array}\right] \\
& {\left[\begin{array}{l}
\mathrm{x}_{\mathrm{f}} \\
\mathrm{y}_{\mathrm{f}} \\
\mathrm{z}_{\mathrm{f}}
\end{array}\right]=\left[\begin{array}{ccc}
c \theta c \psi & s \phi s \theta c \psi-c \phi s \psi & c \phi s \theta c \psi+s \phi s \psi \\
c \theta s \psi & s \phi s \theta s \psi+c \phi c \psi & c \phi s \theta s \psi-s \phi c \psi \\
-s \theta & s \phi c \theta & c \phi c \theta
\end{array}\right]\left[\begin{array}{c}
u \\
v \\
w
\end{array}\right]+\left[\begin{array}{c}
v_{W x_{f}} \\
v_{W f_{f}} \\
v_{W Z_{f}}
\end{array}\right]} \\
& {\left[\begin{array}{c}
\dot{\phi} \\
\dot{\theta} \\
\dot{\psi}
\end{array}\right]=\left[\begin{array}{ccc}
1 & \mathrm{~s} \phi \mathrm{s} \theta / \mathrm{c} \theta & \mathrm{c} \phi \mathrm{s} \theta / \mathrm{c} \theta \\
0 & \mathrm{c} \phi & -\mathrm{s} \phi \\
0 & \mathrm{~s} \phi / \mathrm{c} \theta & \mathrm{c} \phi / \mathrm{c} \theta
\end{array}\right] \cdot\left[\begin{array}{l}
\mathrm{p} \\
\mathrm{q} \\
\mathrm{r}
\end{array}\right]}
\end{aligned}
$$

where:

$\mathrm{u}, \mathrm{v}, \mathrm{w}$ : translational velocity components along the three axes of the body reference frame $\left[\mathrm{m} \mathrm{s}^{-1}\right]$;

p, q, r: rotational velocity components around the three axes of the body reference frame; respectively representing rolling, pitching and yawing rates $\left[\mathrm{rad} \mathrm{s}^{-1}\right]$; 
$\phi, \theta, \psi$ : Euler angles, respectively representing bank, elevation and azimuth/heading Euler rotations [rad];

$\mathrm{x}_{\mathrm{f}}, \mathrm{y}_{\mathrm{f}}, \mathrm{z}_{\mathrm{f}}$ : components of the relative position vector between the Earth-fixed reference frame and the body centre of mass [m]; $\mathrm{F}_{\mathrm{X}_{\mathrm{b}}}, \mathrm{F}_{\mathrm{Y}_{\mathrm{b}}}, \mathrm{F}_{\mathrm{Z}_{\mathrm{b}}}$ : resultants of the aerodynamic and propulsive forces acting along the three axes of the body reference frame $[\mathrm{N}]$;

$\mathrm{M}_{\mathrm{X}_{\mathrm{b}}}, \mathrm{M}_{\mathrm{Y}_{\mathrm{b}}}, \mathrm{M}_{\mathrm{Z}_{\mathrm{b}}}$ : resultants of the aerodynamic and propulsive moments acting around the three axes of the body reference frame $[\mathrm{N} \mathrm{m}]$;

$\mathrm{I}_{\mathrm{XX}_{\mathrm{b}}}, \mathrm{I}_{\mathrm{YY}_{\mathrm{b}}}, \mathrm{I}_{\mathrm{ZZ}_{\mathrm{b}}}, \mathrm{I}_{\mathrm{XZ}_{\mathrm{b}}}$ : non-null components of the inertia tensor $\left[\mathrm{kg} \mathrm{m}^{2}\right]$;

$\mathrm{v}_{\mathrm{WX}_{\mathrm{f}}}, \mathrm{v}_{\mathrm{WY}_{\mathrm{f}}}, \mathrm{v}_{\mathrm{WZ}_{\mathrm{f}}}$ : components of the wind vector along the three axes of the Earth-fixed reference frame $\left[\mathrm{m} \mathrm{s}^{-1}\right]$;

$\mathrm{W}$ : aircraft weight $[\mathrm{N}]$, which may be either constant or subject to fuel consumption. In particular, eqns. (5) to (8) represent the translational dynamics, the rotational dynamics, the kinematics, the Euler rotations of the body frame with respect to the Earthfixed reference frame. Rigid-body models are considered unsuitable for the calculation of trajectories on medium-long timeframes, and this is both due to the large dimensions and complexity of the resulting OCP or estimation problem, as well as the presence of short period modes that are keen to generate numerical instabilities. Rigid body models are nevertheless fundamental for the study of transition manoeuvres and more in general for the dynamic stability and control analysis and design of aircraft, and are successfully adopted in a number of trajectory optimisation studies, in combination with a careful selection of path constraints. A commonly adopted approach, on the other hand, is to derive a simplified set of equations of motion for atmospheric flight based on the approximation of the aircraft as a point-mass object thereby neglecting all aspects associated to its rotational dynamics. The resulting dynamics are characterised by only Three Degrees of Freedom (3-DoF) - i.e. the three spatial coordinates. These models are based on Newton's second law expressed along the coordinate axes of the body frame, and on the expression of the motion of such frame with respect to an inertial reference frame of convenience. As an example, the following set of DAE is associated with a variable mass 3-DoF model:

$$
\left\{\begin{array}{l}
\dot{\mathrm{v}}=\frac{\mathrm{T}-\mathrm{D}}{\mathrm{m}}-\mathrm{g} \sin \gamma \\
\dot{\gamma}=\frac{\mathrm{g}}{\mathrm{v}} \cdot(\mathrm{N} \cos \mu-\cos \gamma) \\
\dot{\chi}=\frac{\mathrm{g}}{\mathrm{v}} \cdot \frac{\mathrm{N} \sin \mu}{\cos \gamma} \\
\dot{\phi}=\frac{\mathrm{v} \cos \gamma \sin \chi+\mathrm{v}_{\mathrm{w}_{\phi}}}{\mathrm{R}_{\mathrm{E}}+\mathrm{z}} \\
\dot{\lambda}=\frac{\mathrm{v} \cos \gamma \cos \chi+\mathrm{v}_{\mathrm{w}_{\lambda}}}{\left(\mathrm{R}_{\mathrm{E}}+\mathrm{z}\right) \cos \phi} \\
\dot{\mathrm{z}}=\mathrm{v} \sin \gamma+\mathrm{v}_{\mathrm{w}_{\mathrm{Z}}} \\
\dot{\mathrm{m}}=-\mathrm{FF}
\end{array}\right.
$$

where the state vector consists of the following variables:

$\mathrm{v}$ : longitudinal velocity (scalar) $\left[\mathrm{m} \mathrm{s}^{-1}\right]$;

$\gamma$ : flight path angle (scalar) [rad];

$\chi$ : track angle (scalar) [rad];

$\phi$ : geographic latitude [rad];

$\lambda$ : geographic longitude $[\mathrm{rad}]$;

z: flight altitude $[\mathrm{m}]$;

$\mathrm{m}$ : aircraft mass $[\mathrm{kg}]$;

and the variables forming the control vector are:

$\mathrm{T}$ : thrust force $[\mathrm{N}]$;

$\mathrm{N}$ : load factor [ ];

$\mu$ : bank angle [rad];

and other variables/parameters include:

$\mathrm{D}$ : aerodynamic drag [N];

$\mathrm{v}_{\mathrm{w}}$ : wind velocity, in its three scalar components $\left[\mathrm{m} \mathrm{s}^{-1}\right]$;

$\mathrm{g}$ : gravitational acceleration $\left[\mathrm{m} \mathrm{s}^{-2}\right]$

$\mathrm{R}_{\mathrm{E}}$ : Earth radius $[\mathrm{m}]$;

FF: fuel flow $\left[\mathrm{kg} \mathrm{s}^{-1}\right]$.

Generally, all aspects associated with the rotational state of the aircraft are neglected and the model can involve either a constant mass or a variable mass. Models belonging to the first category are adopted when the analysed timeframe is relatively limited, so that the fuel consumption may be neglected, or when no fuel is consumed, such as in the case of sailplanes or total engine failures.

\section{Path Constraints}

It is important to note that most of the GNSS integrity degradations depend on the relative position and movements between the GNSS receiver antenna and each satellite independently. Hence degradations will be related to one satellite and will not affect the system with the same manner or intensity. A loss of integrity happens if a combination of several degradations from different satellites happened in the same time. The algorithm and thresholds discussed in $[8,9]$ are precisely capable of detecting combinations of degradations. The system can be summarized as follow, for each satellite of the constellation (32 for the GPS constellation) the algorithm will compute a potential CIF/WIF with the current values of the aircraft flight parameters, the satellite parameters, and the given thresholds. The algorithm will analyze and give an individual WIF and/or a CIF to every satellite of the constellation. Then the number of satellite without CIF and without WIF is evaluated.

A general CIF is triggered is less than 5 satellites are remaining without an individual CIF and general WIF is triggered if less than 4 satellites are remaining without an individual WIF. To be capable of constraining the trajectory optimization process, dynamic constraint criterions need to be set. An analysis of the different type of degradations will show 
that there is no need to set individual criterion for each type of degradation sources, but that a common criterion based on satellite elevation variation in the body frame can be set for all of them. This simplifies and makes possible integrity navigation.

\section{Boundary Conditions}

Boundary conditions define the values that state and control variables of the dynamical system shall have at the initial and final times of the optimisation routine. Since in some instances the boundary conditions are not always restricted to definite values, it is useful to adopt a generalised expression including relaxed conditions. The range has a direct impact on the duration and the result of the optimisation process. The boundary values are determined based on four main sources including:

- Navigation Data and Flight Parameters at CIF (NFC), which define the initial conditions when the optimization process in started (i.e., at CIF generation time step).

- Planned Flight Trajectory (PFT), which define the final condition of the optimization problem. The final condition gives the point when the $\mathrm{A} / \mathrm{C}$ will go back on the initially planned trajectory (e.g., the trajectory stored in the FMS).

- Aircraft Dynamic Constraints (ADC), which define the minimum and maximum values of the state and control variables.

- Satellite Constellation Data (SCD), which provide the azimuth and elevation boundaries for the path constraints.

\section{TRAJECTORY OPTIMISATION FOR MISSION PLANNING AND REAL-TIME APPLICATIONS}

The Radau pseudospectral method is selected for the ABIA mission planning implementation (offline IFG and FPO modules) [13, 14]. Theses widely used methods employ orthogonal collocation with implicit integration based on Gaussian quadrature, where collocation is performed at the Legendre-Gauss-Radau points. For the real-time trajectory generation module implementation, a suitable Direct Constrained Optimisation (DCO) method is implemented. In this method the Aircraft Dynamics Model (ADM) is used to generate a number of feasible flight trajectories that also satisfy the GNSS constraints. The feasible trajectories are calculated by initialising the aircraft dynamics model with a Manoeuvre Identification Algorithm (MIA). The MIA allows identifying a sub-set of ADM equations (and the associated boundary conditions of states and controls) that must be integrated to predict future states and to determine the optimal controls that minimise the cost functions. Although this method does not implement an iterative algorithm that converges to the mathematical optimum, it is preferred for real-time safetycritical applications due to its robustness, much reduced complexity and faster convergence rate. Additionally, the DCO prevents problems of non-convergence or divergence frequently experienced in OCPs for highly non-linear dynamic systems. A schematic of the DCO implementation is shown in Fig. 2.

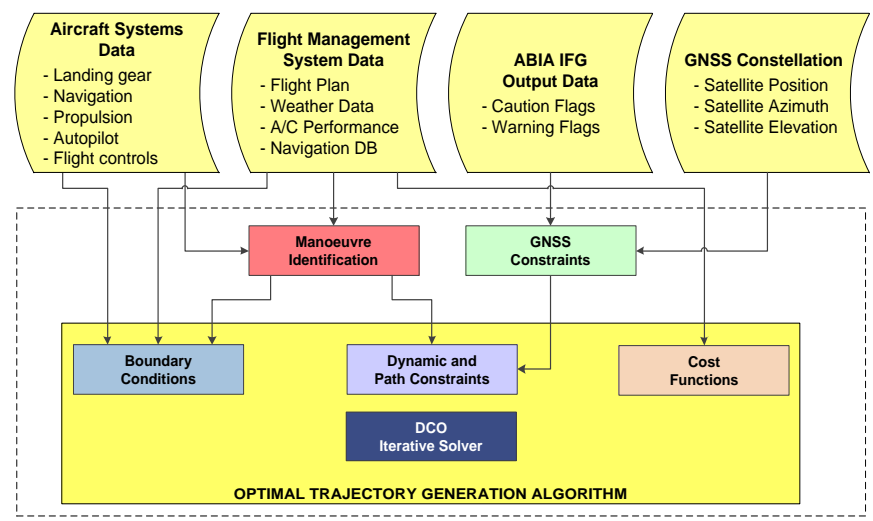

Fig. 2. DCO implementation scheme.

In both real-time and mission-planning implementations, the flight trajectory optimisation algorithm is initiated when integrity degradations are predicted (CIF generated) by the IFG module. The optimisation criteria adopted in the FPO module are the following:

- With 5 satellites in view, the value of elevation angle for each satellite tracked shall be 5 degrees greater than the threshold value causing the activation of any CIF.

- With 4 satellites in view, the value of elevation angle for each satellite tracked shall be 10 degrees greater than the threshold value causing the activation of any CIF.

- When the $\mathrm{C} / \mathrm{N}_{0}$ is less than $26 \mathrm{~dB}-\mathrm{Hz}$, the $\mathrm{CIF}$ shall be generated.

- When the $\mathrm{C} / \mathrm{N}_{0}$ is less than $25 \mathrm{~dB}-\mathrm{Hz}$, the WIF shall be generated.

- When the $\mathrm{C} / \mathrm{N}_{0}$ is below $28 \mathrm{~dB}-\mathrm{Hz}$ and the signal is lost, the caution integrity flag shall be generated if the estimated acquisition time is less than the application-specific TTA requirements.

- When the $\mathrm{C} / \mathrm{N}_{0}$ is below $28 \mathrm{~dB}-\mathrm{Hz}$ and the signal is lost, the warning integrity flag shall be generated if the estimated acquisition time exceeds the application-specific TTA requirements.

- Constrained Geometric Optimisation (CGO) or PseudoSpectral Optimisation (PSO) techniques are used for real-time FPO implementations and for offline missionplanning applications respectively.

\section{COST FUnCTIONS}

The selection of the optimal trajectory is based on minimising a cost function of the form [15]: 


$$
J=w_{t} \cdot t+w_{f} \int[S F C \cdot T(t)] d t
$$

where SFC $\left[\mathrm{kg} \mathrm{N}^{-1} \mathrm{~s}\right]$ is the specific fuel consumption, $\mathrm{T}(\mathrm{t})$ is the thrust profile and $\left\{\mathrm{w}_{\mathrm{t}}, \mathrm{w}_{\mathrm{f}}\right\}$ are the weightings attributed to time and fuel minimisation objectives. In safety-critical applications, this cost function can be expanded to include other parameters such as the distance of the host aircraft from the avoidance volume associated with an obstacle or a conflicting air traffic [16] and is modified as:

$$
\begin{gathered}
J=w_{t} \cdot t_{\text {SAFE }}+w_{f} \int[S F C \cdot T(t)] d t \\
-w_{d} \cdot D_{\min }+-w_{i d} \cdot \int D(t) d t
\end{gathered}
$$

where $\mathrm{D}(\mathrm{t})$ is the estimated distance of the generated avoidance trajectory points from the avoidance volume associated with the obstacle, $D_{\min }=\min [D(t)]$ is the estimated minimum distance of the avoidance trajectory from the avoidance volume, $\mathrm{t}_{\mathrm{SAFE}}=$ $\left.t\right|_{D_{\min }}$ is the time at which the safe avoidance condition is successfully attained and $\left\{\mathrm{w}_{\mathrm{t}}, \mathrm{w}_{\mathrm{f}}, \mathrm{w}_{\mathrm{d}}, \mathrm{w}_{\mathrm{id}}\right\}$ are the weightings attributed to time, fuel, distance and integral distance respectively.

\section{Simulation Case STUdy}

In order to validate the design of the ABIA IFG module and the trajectory optimisation algorithms, MATLAB ${ }^{\circledR}$ simulation case studies were performed. An AIRBUS A320 3-DoF ADM is used as a reference for the simulation case study. All data for the Airbus 320 aircraft are obtained from Eurocontrol BADA database [15]. This is a collection of data, which specifies operation performance parameters for a certain number of different aircraft. The modeling was performed with models from the BADA user manual [15]. The A320 STereoLithograph (STL) model was used in the simulation case studies and is shown in Fig. 3.

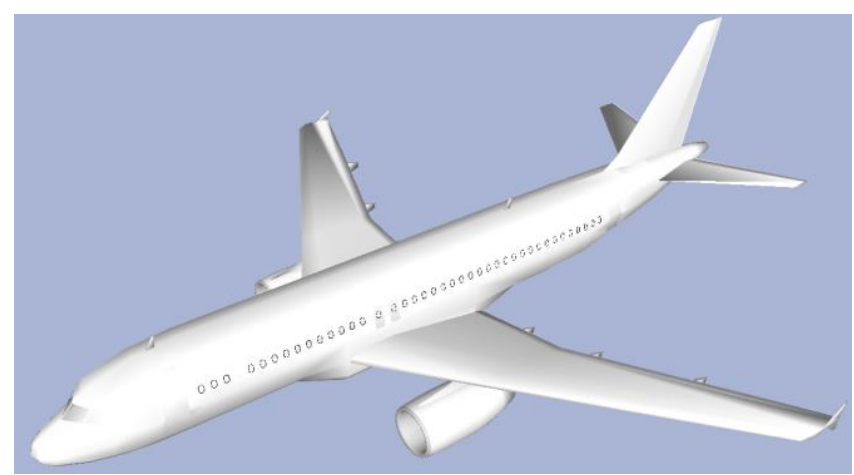

Fig. 3. STL model of AIRBUS A320 aircraft.

The Global Positioning System (GPS) constellation was simulated and the GNSS receiver tracking loops were modelled with a flat random vibration power curve from $20 \mathrm{~Hz}$ to $2000 \mathrm{~Hz}$ with amplitude of $0.005 \mathrm{~g}^{2} / \mathrm{Hz}$ and the oscillator vibration sensitivity $S_{v}\left(f_{m}\right)=1 \times 10^{-9}$ parts/g. An initial trajectory was used as a reference and was obtained by using the A320 3-DoF ADM. The simulated AIRBUS A320 trajectory included six

\begin{tabular}{|c|c|c|c|c|c|}
\hline States & Speed & V & $=$ & 130 & {$[\mathrm{~m} / \mathrm{s}]$} \\
\hline & FPA & $\gamma$ & $=$ & 0 & {$\left[{ }^{\circ}\right]$} \\
\hline & Heading & $\psi$ & $=$ & 270 & {$\left[{ }^{\circ}\right]$} \\
\hline & Mass & $m$ & $=$ & 70000 & {$[\mathrm{~kg}]$} \\
\hline & Latitude & $\varphi$ & $=$ & 0.9 & {$\left[{ }^{\circ}\right]$} \\
\hline & Longitude & $\theta$ & $=$ & -0.008 & {$\left[{ }^{\circ}\right]$} \\
\hline & Altitude & $h$ & $=$ & 50 & [m] \\
\hline & Leg 1 & & & & \\
\hline Controls & Pitch & $\Gamma$ & $=$ & 20 & {$\left[{ }^{\circ}\right]$} \\
\hline & Bank & $\phi$ & $=$ & 0 & {$\left[{ }^{\circ}\right]$} \\
\hline & Throttle & $T h$ & $=$ & Take-off/Climb & {$[-]$} \\
\hline & Leg 2 & & & & \\
\hline Controls & Pitch & $\Gamma$ & $=$ & 10 & {$\left[{ }^{\circ}\right]$} \\
\hline & Bank & $\phi$ & $=$ & 25 & {$\left[{ }^{\circ}\right]$} \\
\hline & Throttle & $T h$ & $=$ & Take-off/Climb & {$[-]$} \\
\hline & Leg 3 & & & & \\
\hline Controls & Speed & $V$ & $=$ & 230 & {$[\mathrm{~m} / \mathrm{s}]$} \\
\hline & Pitch & $\Gamma$ & $=$ & 0 & {$[\circ]$} \\
\hline & Bank & $\phi$ & $=$ & 0 & {$\left[{ }^{\circ}\right]$} \\
\hline & Throttle & $T h$ & $=$ & Cruise & {$[-]$} \\
\hline & Leg 4 & & & & \\
\hline Controls & Pitch & $\Gamma$ & $=$ & 0 & {$[\circ]$} \\
\hline & Bank & $\phi$ & $=$ & 50 & {$\left[{ }^{\circ}\right]$} \\
\hline & Throttle & $T h$ & $=$ & Cruise & [RPM] \\
\hline & Leg 5 & & & & \\
\hline Controls & Speed & V & $=$ & 130 & {$[\mathrm{~m} / \mathrm{s}]$} \\
\hline & FPA & $\gamma$ & $=$ & -5 & {$[\circ]$} \\
\hline & Pitch & $\Gamma$ & $=$ & -5 & {$[\circ]$} \\
\hline & Bank & $\phi$ & $=$ & -40 & {$[\circ]$} \\
\hline & Throttle & $T h$ & $=$ & Descent/Landing & {$[-]$} \\
\hline & Leg 6 & & & & \\
\hline Controls & Pitch & $\Gamma$ & $=$ & -5 & {$\left[{ }^{\circ}\right]$} \\
\hline & Bank & $\phi$ & $=$ & 0 & {$[\circ]$} \\
\hline & Throttle & $T h$ & $=$ & Descent/Landing & {$[-]$} \\
\hline
\end{tabular}
flight phases, whose parameters as provided in Table I.

Table I. Simulation parameters. 
The six flight phases used for simulation include:

- $\quad$ Climb phase (0-300s);

- $\quad$ Turning climb phase (300-600s);

- $\quad$ Straight and level (cruise) phase (600-900s);

- $\quad$ Level turn phase (900-1200s)

- $\quad$ Turning descend phase (1200-1500s);

- $\quad$ Descend (straight approach) phase (1500-1800s);

The resultant scenario is shown in Fig. 4 with the six flight phases highlighted distinctively.

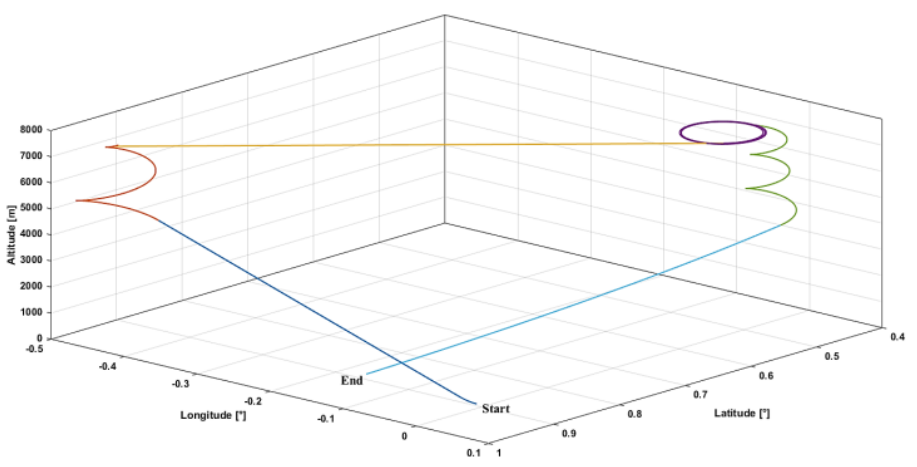

Fig. 4. Simulation scenario.

All CIFs and WIFs relative to antenna masking, geometric accuracy degradations, SNR, multipath and Doppler shift were generated. In some cases, the CIF was generated but it was not followed by the WIF (this was due to a temporary adverse relative geometry not leading to GNSS signal losses). During the level turn and turning descent phases, the CIFs were followed by the WIF. It was also observed that the CIF was always triggered at least 2 seconds before the successive WIF onset. The CIFs and WIFs generated for all flight phases are shown in Fig. 5.

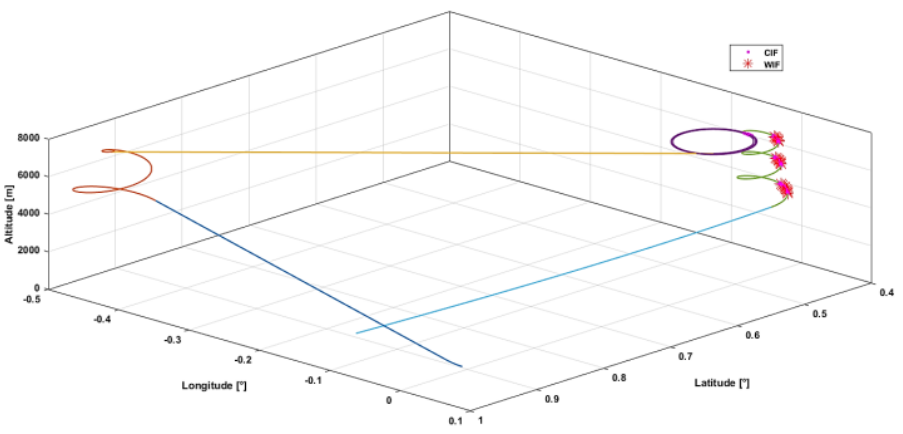

Fig. 5. Generation of CIFs and WIFs.

The results corroborate the validity of the models developed for the CIF/WIF thresholds. It is evident that the availability of a usable CIF represents a significant progress in this research with the potential for both manned and unmanned aircraft to recover from mission- and safety-critical flight conditions potentially leading to GNSS data losses. Therefore, it is envisaged that a properly designed ABIA FPO module will take full advantage of this predictive behaviour, allowing the aircraft to correct its flight trajectory/attitude in order to avoid the occurrence of the critical GNSS data losses. Additionally, it is possible that this predictive behaviour can be exploited in the pursuit of a GNSS based autolanding capability. In order to evaluate the trajectory optimisation algorithms, a segment in level turn phase is considered as shown in Fig. 6.

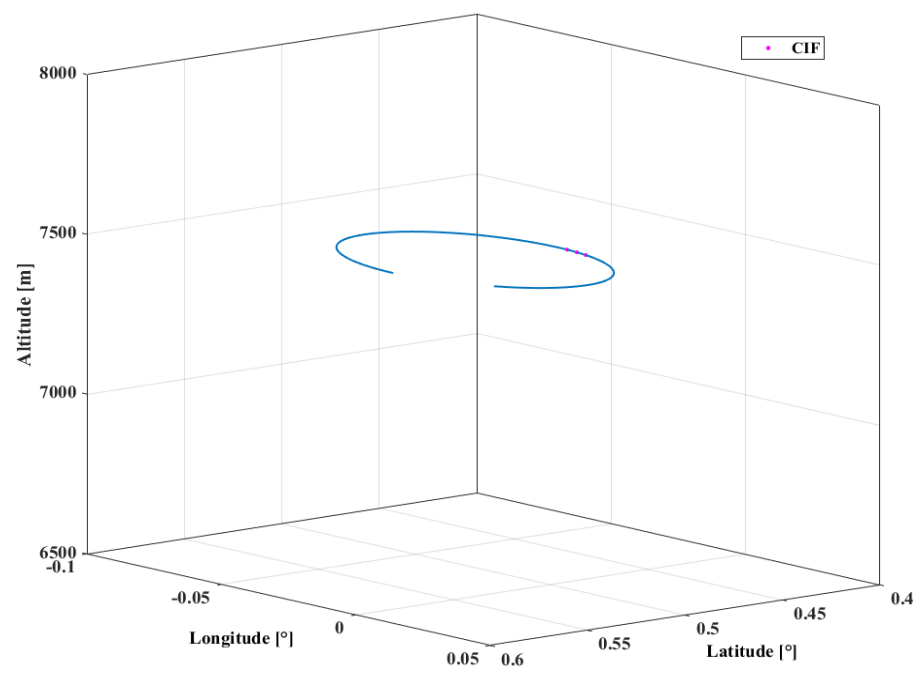

Fig. 6. Selected segment for optimisation.

In this particular segment of level turn flight phase, the satellites constellation situation at the first CIF time step expressed in the body frame. The bank angle in this case is $50^{\circ}$. This is a quite steep, especially for an airliner, but the selected aircraft is capable of flying such a manoeuvre according to its design constraints. Considering the first CIF time state vector as an initial guess, a re-optimised trajectory is generated to avoid the occurrence of any CIF/WIF. The optimised segment obtained by using PSO technique free of any integrity flags is shown in Fig. 7.

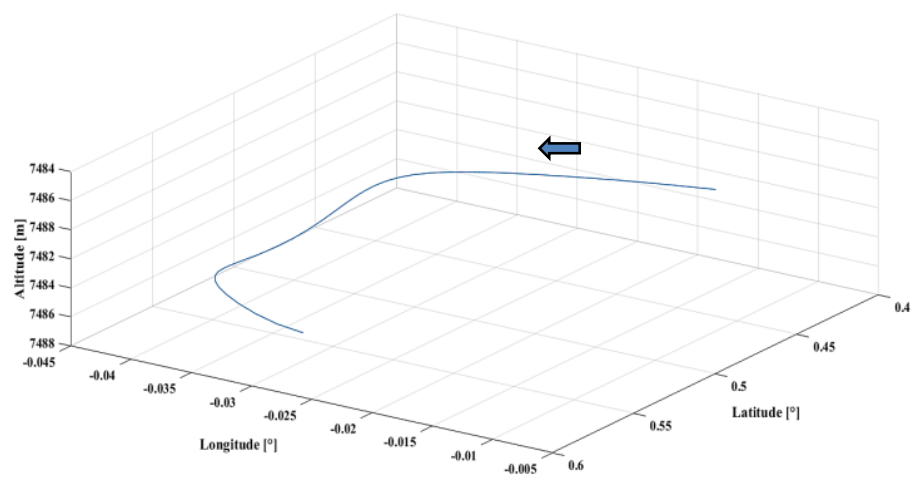

Fig. 7. Optimised segment free of integrity flags. 
To avoid degradations and at the same time to satisfy the path constraints with the conditions set on satellite elevation angles, the optimised trajectory is obtained by decoupling the pitch and the bank angles. It was observed that before optimisation, the elevation drops significantly after the first integrity degradation prediction, which leads to an integrity loss. After optimisation, it was observed that the elevation angle is high enough to prevent the occurrence of CIF/WIF. The new trajectory planning algorithm is then run with all the parameters specified in Table I. The variation of state variables during the optimisation of the selected flight segment is shown in Fig. 8. In general, the PMO technique converged to a mathematical optimum within 5 - 220 seconds depending on the length and complexity of the flight path to be optimised, while the CGO technique generated trajectories free from GNSS data degradations/losses within 0.3 0.9 seconds.
The results provide evidence that the PMO algorithms cannot be directly employed in real-time ABIA applications. This is because, considering the flight dynamics of A320 and other airliners, the time required to perform trajectory optimisation is too long for real-time path following tasks in Automatic Flight Control System (AFCS). As already mentioned, the PMO technique is capable of generating a mathematical optimum and is better suited for ABIA mission-planning/ATM applications (i.e., ground-based and avionics mission planning tools). It is therefore concluded that the adoption of PMO/CGO techniques in the ABIA FPG module would allow an efficient exploitation of the IFG module predictive features both in mission planning and real-time trajectory optimisation problems. Therefore, it offers the potential to meet the GNSS integrity requirements for ATM online operations and AFCS/ABIA integration for GLS down to CAT II/III.
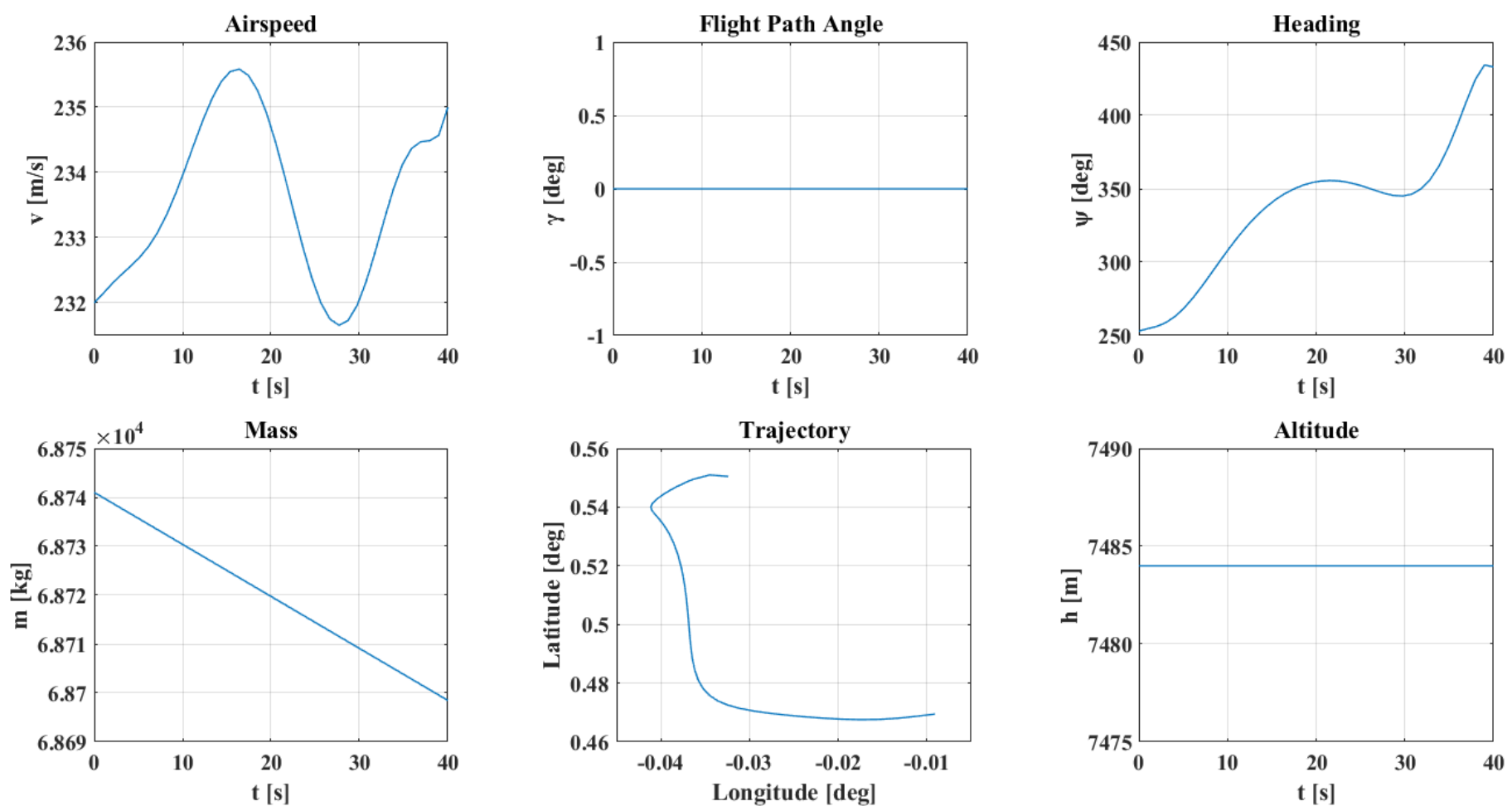

Fig. 8. Trajectory optimisation state variables.

\section{CONCLUSIONS AND FUTURE WORK}

The trajectory optimisation algorithms for flight planning and real-time optimisation algorithms adopted in the GNSS Avionics Based Integrity Augmentation (ABIA) system were presented. In case of flight planning, a Pseudo Spectral Optimisation (PSO) technique was adopted, while for the real-time optimisation implementation, a Direct Constrained Optimisation (DCO) method is preferred. A detailed simulation case study was performed for the ABIA integrity flag generator and trajectory optimisation algorithms using the AIRBUS A320 aircraft dynamics model. According to the results obtained from modeling and simulation activities, after the integrity caution flag is generated, the time available for the pilot/remote pilot/autopilot to react (before the integrity warning flag is generated), is sufficient for safety-critical tasks including GLS curved/segmented precision approach and automatic landing applications. Further research is focusing on the following areas: 
- Extend the ABAS/ABIA concept to other navigation, communication and surveillance applications in the Communication, Navigation, Surveillance/Air Traffic Management and Avionics (CNS+A) context.

- Investigate the potential of ABIA to enhance the performance of Next Generation Flight Management Systems (NG-FMS) for manned and unmanned aircraft [16].

- Evaluate the potential of ABAS/ABIA techniques to enhance the performance of next generation $\mathrm{CNS}+\mathrm{A}$ decision support tools for Performance/Intent Based Operations (PBO/IBO) and four dimensional trajectory management [17].

- Investigate the potential of ABAS/ABIA concepts to support aviation forensic applications (i.e., accident and incident investigation).

- Assess the potential synergies between ABIA and RAIM techniques, including enhanced RAIM (eRAIM) and predictive RAIM (pRAIM) in a multi-constellation GNSS environment.

- Further investigate the potential of ABAS/ABIA techniques to support safety-critical applications such as Sense-andAvoid (SAA) in the UAS Traffic Management (UTM) context [18].

- Investigate the potential of ABAS/ABIA techniques to enhance GNSS integrity in low-cost integrated navigation and guidance systems for autonomous operations [19-21].

\section{REFERENCES}

[1] SESAR Joint Undertaking, "SESAR Release", no. 2, March 2012

[2] FAA, "The Future of the NAS", US Department of Transportation, Federal Aviation Administration, Washington DC, USA, 2016.

[3] J.M.C. Rodriguez, L.G. Deniz, J. G. Herrero and J. B. Portas, "A 4D Trajectory Negotiation Protocol for Arrival and Approach Sequencing," Integrated Communications, Navigation and Surveillance Conference (ICNS 2008), Bethesda, MD, USA, 2008.

[4] European Organisation for the Safety of Air Navigation, "Initial 4D Trajectory Data Link (4DTRAD) Concept of Operations", Eurocontrol, December 2008.

[5] K. Chircop, D. Zammit-Mangion and R. Sabatini, "Bi-Objective Pseudospectral Optimal Control Techniques for Aircraft Trajectory Optimization," 28th Congress of the International Council of the Aeronautical Sciences 2012, ICAS 2012, vol. 5, pp. 3546-3555, Brisbane, Australia, 2012.

[6] ICAO, Doc 9613 - Performance-based Navigation (PBN) Manual, The Internaltional Civil Aviation Organization, Third edition, Montreal, Canada, 2008.
[7] R. Sabatini, "Differential Global Positioning System (DGPS) for Flight Testing," NATO Research and Technology Organization (RTO) - Systems Concepts and Integration Panel (SCI). AGARDograph Series RTO-AG160 , vol. 21, Oct 2008 .

[8] R. Sabatini, T. Moore, and C. Hill, "A New Avionics Based GNSS Integrity Augmentation System: Part 1 - Fundamentals," Journal of Navigation, vol. 66, no. 3, pp. 363-383, May 2013. DOI: $10.1017 / \mathrm{S} 0373463313000027$

[9] R. Sabatini, T. Moore, and C. Hill, "A New Avionics Based GNSS Integrity Augmentation System: Part 2 - Integrity Flags," Journal of Navigation, vol. 66, no. 4, pp. 511-522, June 2013. DOI: $10.1017 / \mathrm{S} 0373463313000143$

[10] A. Gardi, R. Sabatini and S. Ramasamy, "Multi-Objective Four Dimesional Trajectory Optimisation in the ATM Context," Progress in the Aerospace Sciences, vol. 83, pp. 1-36. DOI: 10.1016/j.paerosci.2015.11.006

[11] D. G. Hull, Fundamentals of airplane flight mechanics, Springer-Verlag, Berlin/Heidelberg, Germany, 2010.

[12] W. F. Phillips, Mechanics of Flight, $2^{\text {nd }}$ ed., John Wiley and Sons, Hoboken, NJ, USA, 2010.

[13] A. V. Rao, "Survey of Numerical Methods for Optimal Control," Advances in the Astronautical Sciences, vol. 135, pp. 497-528, 2010

[14] M. P. Hansen, "Tabu Search Multiobjective Optimisation: MOTS," $13^{\text {th }}$ International Conference on Multiple Criteria Decision Making (MCDM '97), Cape Town, South Africa, 1996

[15] Eurocontrol, "User Manual for the Base of Aircraft Data (BADA) Revision 3.11", Eurocontrol Experimental Centre (EEC) Technical/Scientific Report No. 13/04/16-01, Brétigny-sur-Orge, France, 2013.

[16] S. Ramasamy and R. Sabatini, "A Unified Approach to Cooperative and Non-Cooperative Sense-and-Avoid," International Conference on Unmanned Aircraft Systems (ICUAS 2015), Denver, CO, USA, 2015.

[17] A. Gardi, R. Sabatini, S. Ramasamy and T. Kistan, "Real-Time Trajectory Optimisation Models for Next Generation Air Traffic Management Systems", Applied Mechanics and Materials, vol. 629, pp. 327-332, 2014. DOI: 10.4028/www.scientific.net/AMM.629.327

[18] S. Ramasamy, R. Sabatini and A. Gardi, "LIDAR Obstacle Warning and Avoidance System for Unmanned Aerial Vehicle Sense-and-Avoid", Aerospace Science and Technology, vol. 55, pp. 344-358, 2016. DOI: 10.1016/j.ast.2016.05.020

[19] R. Sabatini, L. Rodriguez, A. Kaharkar, C. Bartel and T. Shaid, "CarrierPhase GNSS Attitude Determination and Control System for Unmanned Aerial Vehicle Applications", ARPN Journal of Systems and Software, vol. 2, no.11, pp. 297-322, 2012.

[20] R. Sabatini, C. Bartel, A. Kaharkar, T. Shaid and L. Rodriguez, "Low-cost Navigation and Guidance Systems for Unmanned Aerial Vehicles - Part 1: Vision-based and Integrated Sensors, Annual of Navigation, vol. 19, no. 2, pp. 71-98, 2013.

[21] S. Bijjahalli, S. Ramasamy and R. Sabatini, "A GNSS Integrity Augmentation System for Sustainable Autonomous Airside Operations," $2^{\text {nd }}$ International Symposium on Sustainable Aviation (ISSA 2016), Istanbul, Turkey, 2016. 